\title{
Avaliação de um programa de curso de Medicina utilizando o módulo lógico
}

\author{
Evaluation of a Medical course program using the logic module \\ Evaluación del programa de un curso de Medicina utilizando el módulo lógico
}

Recebido: 18/01/2022 | Revisado: 22/01/2022 | Aceito: 22/01/2022 | Publicado: 24/01/2022

\author{
Stefânia Moreira de Paula \\ ORCID: https://orcid.org/0000-0002-5503-9215 \\ Universidade Federal de São João de-Rei, Brasil \\ E-mail: stefania_moreira07@outlook.com \\ Laila Cristina Moreira Damázio \\ ORCID: https://orcid.org/0000-0001-7370-8892 \\ Universidade Federal de São João del-Rei, Brasil \\ Universitário Presidente Tancredo de Almeida Neves, Brasil \\ E-mail: lailacmdamazio@gmail.com
}

\begin{abstract}
Resumo
O presenta artigo apresenta avaliação de programas educacionais, mais precisamente o modelo lógico de avaliação, Context a Input a Process a Product (CIPP), para verificar a qualidade do ensino-aprendizagem do curso de medicina de uma instituição pública no interior de Minas Gerais. Dessa forma, o estudo teve como objetivo avaliar o programa educacional do curso de medicina de uma instituição pública do interior de Minas Gerais utilizando o instrumento de Modelo Lógico ou CIPP. Para isso, foi realizada análise da Diretriz Curricular Nacional (DCN) dos Cursos de Medicina, o Projeto Pedagógico do Curso (PPC), as atas do Núcleo Docente Estruturante (NDE) e posteriormente foi realizado levantamentos de dados das entrevistas com os técnicos, discente e docentes do curso. O modelo lógico analisou o programa do curso de medicina apontando para questões que promovem o melhor ensino-aprendizado dos discentes e questões estruturais que necessitam ser melhoradas no município e na instituição de ensino superior.
\end{abstract}

Palavras-chave: CIPP; Ensino-aprendizagem; Diretriz curricular nacional; Projeto pedagógico do curso; Núcleo docente estruturante.

\begin{abstract}
This article presents the evaluation of educational programs, more precisely the logical evaluation model, Context a Input a Process a Product (CIPP), to verify the quality of teaching and learning in the medical course of a public institution in the interior of Minas Gerais. Thus, the study aimed to evaluate the educational program of the medical course of a public institution in the interior of Minas Gerais using the Logical Model or CIPP instrument. For this, an analysis was carried out of the National Curriculum Guidelines (DCN) of Medicine Courses, the Pedagogical Project of the Course (PPC), the minutes of the Structuring Teaching Nucleus (NDE) and later data surveys were carried out from the interviews with the technicians, student and course teachers. The logical model analyzed the medical course program pointing to issues that promote better teaching-learning for students and structural issues that need to be improved in the municipality and in the higher education institution.
\end{abstract}

Keywords: CIPP; Teaching-learning; National curriculum guidelines; Pedagogical project of the course; Structuring teaching nucleus.

\section{Resumen}

Este artículo presenta la evaluación de programas educativos, más precisamente el modelo de evaluación lógica, Contexto a Insumo a Proceso a Producto (CIPP), para verificar la calidad de la enseñanza y el aprendizaje en la carrera de medicina de una institución pública del interior de Minas Gerais. Así, el estudio tuvo como objetivo evaluar el programa educativo del curso de medicina de una institución pública del interior de Minas Gerais utilizando el Modelo Lógico o instrumento CIPP. Para ello se realizó un análisis de las Directrices Curriculares Nacionales (DCN) de las Carreras de Medicina, el Proyecto Pedagógico de la Carrera (PPC), las actas de los Núcleos Docentes Estructurantes (NDE) y posteriormente se realizaron levantamientos de datos a partir de las entrevistas. con los técnicos, alumnos y profesores del curso. El modelo lógico analizó el programa de la carrera de medicina señalando aspectos que favorecen una mejor enseñanza-aprendizaje de los estudiantes y aspectos estructurales que necesitan ser mejorados en el municipio y en la institución de educación superior.

Palabras clave: CIPP; Enseñanza-aprendizaje; Lineamientos curriculares nacionales; Proyecto Pedagógico de la carrera; Núcleo docente estructurante. 


\section{Introdução}

Avaliação de programas na área da saúde são mecanismos capazes de abordar, de forma sistemática, a análise e interpretação de aspectos de programas educacionais visando a relevância para a sociedade. (Goldie, 2006). Esse mecanismo possibilita adentrar dentro do curso de medicina para averiguar as práticas educacionais exercidas pelos professores dentro das disciplinas ou um conjunto de disciplinas de uma mesma área até o currículo do curso como um todo (Bollela e Castro, 2014).

O curso de medicina é tido como um dos cursos que mais necessita do empenho de seus estudantes, visto que esses precisam de inteira dedicação para tornar-se um bom profissional. Dessa forma, a avaliação de programas torna-se inteiramente importante para conduzir e averiguar a eficiência dos discentes, bem como o número de egressos elevados, uma vez que é perceptível o aumento do número de instituições abertas que visam formar médicos no Brasil. Ademais, é importante ressaltar que a instituição formadora tem responsabilidade com a sociedade, formando alunos de inteira competência para lidar com o ser humano, bem como demonstrar, não somente a habilidade e o bom treinamento recebido na graduação e pós-graduação, mas também oferecer à atenção devida para seus pacientes considerando a humanização em saúde como uma competência importante na área da saúde (Perim et al., 2005, Mezzalira et al., 2022).

É importante destacar que a avaliação de programas compreende dois tipos de avaliação, a avaliação interna e externa. A avaliação interna é de iniciativa da própria instituição visando a melhoria gradual dos programas educacionais. A avaliação externa pode ser entendida como uma própria opção da instituição em convidar/contratar especialistas para ter acesso aos documentos, aos profissionais, com ou sem visitas in loco para revisarem ou recomendarem novas abordagens de ensino/aprendizagem. Os pareceres podem ressaltar a necessidade de melhoria de novas práticas ou colocar em prática novos métodos de ensino reorientando os gestores do curso (Bollela e Castro, 2014).

Há inúmeras razões para a instituição preocupar-se com a formação de profissionais na área da saúde principalmente para lidarem, de forma bem-sucedida, com a sociedade (Haji et al., 2013). Por essa razão, o instrumento de avaliação de programas denominado, Modelo Lógico ou Modelo de Contexto, de Insumos, de Processos e de Produtos ou em inglês Context a Input a Process a Product (CIPP) é um dos modelos conceituais de avaliação de programas que se enquadra no modelo lógico que é comumente utilizado para planejamentos e avaliações de programas, apresentando uma estrutura racional para todas as etapas observadas no processo avaliativo. É caracterizado por ser um modelo simples e linear que apresenta como um dos seus componentes as perguntas que devem ser feitas em cada etapa da avaliação, abordando o contexto, os recursos necessários, o processo e o produto da avaliação (Frye e Hemmer, 2012).

O CIPP pode ser uma ferramenta importante para constituirumpoderoso meio de trabalho dos objetivos educativos, pois colabora para informar e analisar a qualidade dos cursos de medicina, bem como a formação de estudantes da área de saúde, uma vez que esses se tornarão responsáveis pelas decisões tomadas no exercício da profissão (Frye e Hemmer, 2012). E, para que se tenha êxito no trabalho, desde já, torna-se um fator primordial para avaliação dos programas de saúde da instituição, de forma que, paulatinamente, melhore, cada vez mais, o ensino/aprendizagem, alimentando a tomada de decisão e proporcionando melhores práticas médicas (Haji et al., 2013).).

Segundo Frye e Hemmer (2012), avaliar de forma rigorosa e defensável é valorizar as informações que possibilitam adequação e melhoria contínuas nos processos educacionais, pois o aprendizado bem estruturado significa boa formação de profissionais que atendam à sociedade. Por isso, a avaliação de programas é um objeto de estudo primordial para se alcançar a eficácia do ensino/aprendizagem e consequentemente obter um bom desempenho profissional dos seus egressos.

O objetivo dos programas de avaliações educacionais é fazer com que as instituições busquem melhorias para o ensino de seus alunos e uma boa formação acadêmica (Goldie, 2006). É de inteira relevância, também, difundir o programa dentro dos ensinos superiores, uma vez que a avaliação de programas necessita de mecanismos estruturados e regulares para o credenciamento e recredenciamento de cursos superiores (Sinaes, 2010). Além disso, é pouco divulgada e, ao mesmo tempo, é 
de extrema importância, pois visa garantir altos padrões de qualidade para programas que formam profissionais da área da saúde, pois a sociedade exige, por direito, bons atendimentos médicos e com boa formação acadêmica, tendo como produto final um foco maior no processo de aprendizagem (Bollela e Castro, 2014).

O objetivo do presente estudo foi avaliar o programa educacional do curso de medicina de uma instituição pública do interior de Minas Gerais utilizando o instrumento de Modelo Lógico ou CIPP.

\section{Metodologia}

A pesquisa foi realizada no período de julho de 2019 a julho de 2020 no curso de medicina da instituição pública do interior de Minas Gerais. O estudo é do tipo qualitativo com análise documental e as respostas das entrevistas realizadas com os técnicos, discentes e docentes. No curso de medicina ministram aulas, 55 (cinquenta e cinco) docentes; existem 7 (sete) técnicos administrativos e 12 turmas de medicina com entrada de 20 (vinte) alunos em cada turma, totalizando 240 (duzentos e quarenta) alunos.

Para a realização deste estudo foi utilizado o Modelo Lógico ou CIPP que é um instrumento de avaliação de programas confiável e validado no Brasil.

O Modelo Lógico ou CIPP é um instrumento de avaliação de programas com a função de avaliação para tomada de decisões. Sendo que, ele possui fases que antecedem a atividade avaliativa, como $o$ Planejamento (o que se deseja fazer?), $a$ estruturação (como se deseja fazer?), a implementação (será que se está a fazer tal e como foi planejado? Se não, por quê?) e $a$ revisão (será que está a funcionar adequadamente? Se não, por quê?). Sendo que, cada fase possui quatro avaliações: de Contexto, de Insumos, de Processos e de Produtos, denominação para as iniciais do CIPP (Andriola, 2010).

Na Figura 1 está representado os aspectos essenciais de Modelo Lógico ou CIPP que foi aplicado no processo de avaliação do programa educacional do curso de medicina. 
Figura 1. Modelo Lógico ou CIPP.

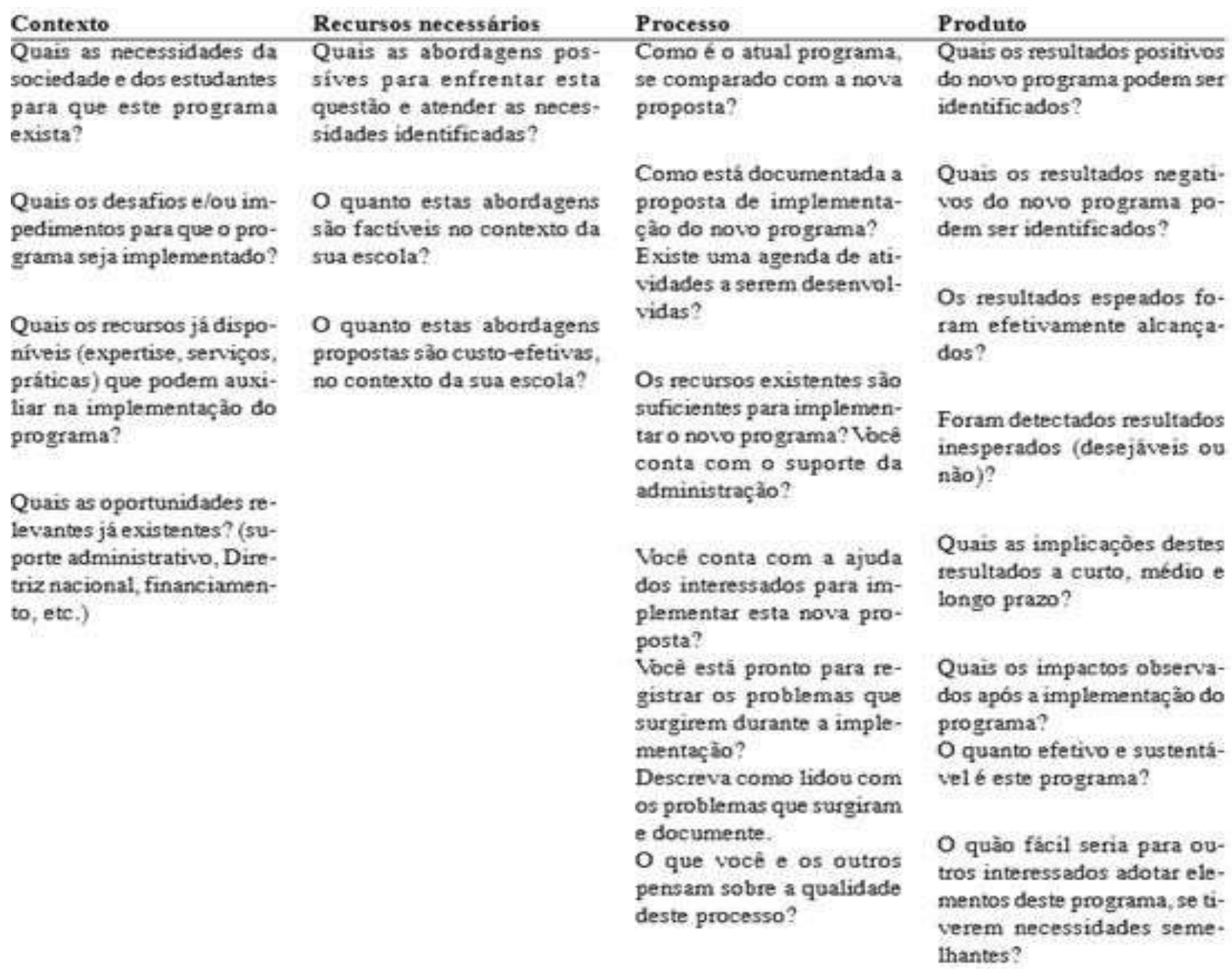

Fonte: Adaptado de Frye e Hemmer (2012) e Bollela e Castro (2014).

Para aplicação do modelo lógico foi realizada análise dos PPC's do curso de medicina, o antigo e o atual. Foi realizada análise da qualificação do corpo docente e de técnicos. Foi realizada análise da infraestrutura física considerando os instrumentos de avaliação externa do Instituto Nacional de Estudos e Pesquisas Educacionais Anísio Teixeira (INEP) Sistema Nacional de Avaliação da Educação Superior (Sinaes).

Foi realizada análise se o PPC contempla as DCNs de Medicina (2014) e se as metodologias de ensinoaprendizagem do curso estão de acordo com o documento norteador da área no Brasil.

Foram realizadas análises das atas do Núcleo Docente Estruturante (NDE) e colegiado do curso desde a sua implantação até o momento. Foram elaboradas entrevistas estruturais para aplicação entre os técnicos, discentes e docentes do curso. As entrevistas foram estruturadas com perguntas discursivas para que o entrevistado tivesse a liberdade de responder o que achava pertinente.

Os dados foram analisados de forma qualitativa de acordo com a análise documental e das respostas nas entrevistas. O projeto foi submetido ao Comitê de Ética envolvendo Seres Humanos com o número de CAAE de 29577820.2.0000.5151.

\section{Resultados e Discussão}

Ao embasar este trabalho no Modelo Lógico de avaliação CIPP, um modelo que envolve, não somente a avaliação, mas também o planejamento do curso, procurou-se averiguar se o PPC estava de acordo com as DCNs de Medicina (2014), já que o curso de medicina é relativamente novo. Foi analisado se o perfil proposto no PPC na implantação do curso, em 2014, 
estava de encontro com as questões pontuadas na DCN (2014) de Medicina. Também foram consideradas as atas do NDE e colegiado do curso para compreensão do desenvolvimento, planejamento, decisões e organizações desempenhadas pelos membros do núcleo docente. Por fim, foram realizadas análises das metodologias ativas praticadas no curso com base no PPC, ressaltando aquela em que traz mais dinamicidade no aprendizado dos alunos para, futuramente, serem aptos às tomadas de decisões na comunidade atuante.

Os resultados destas análises foram comprovados ao longo das descrições feitas posteriormente na abordagem do PPC, da DCN, das atas da NDE e da entrevista feita com os membros participantes. Para isso, foram abordados itens que permitissem a compreensão da estruturação de todo o curso, como pode ser evidenciado abaixo.

\subsection{Projeto Pedagógico do Curso de Medicina e a Diretriz Curricular Nacional de Medicina no Brasil (2014)}

Segundo o portal do Ministério da Educação (MEC), a DCN é um conjunto de regras que orientam a implementação do planejamento curricular das escolas e instituições de ensino. São normas que dão estrutura para o desenvolvimento deste planejamento e é usado pelas instituições como base de seu programa. Elas tiveram um papel de regulamentação e estruturação para o currículo da educação básica e ensino médio, dando a eles mais inovação e transformação na educação, além de ter surgida a partir da Lei de Diretrizes e Bases da Educação (LDB) que

“...assinala ser incumbência da União 'estabelecer, em colaboração com os estados, Distrito Federal e os municípios, competências e diretrizes para a Educação Infantil, o Ensino Fundamental e o Ensino Médio, que nortearão os currículos e os seus conteúdos mínimos, de modo a assegurar a formação básica comum'". (Educação, 2018).

Nas DCN's, como marco de uma estrutura que orienta a construção de um projeto de curso, devem ser respeitadas, logo, o PPC deve estar de acordo com as diretrizes. Dessa forma, o projeto do curso de medicina se enquadrou nas DCNs propostas pelo governo federal, constatando em:

“As DCNs de 2014 (MEC, 2014) foram os marcos teóricos na construção do PPC do curso de Medicina, o qual está voltado para a dimensão da saúde coletiva e gestão, para o aperfeiçoamento do SUS, e para a formação de profissionais competentes, críticos, comprometidos com a organização da assistência e a busca de maiores níveis de responsabilidade institucional.” (Rei, 2018)

Para demonstrar que o curso de medicina segue a DCN proposta pelo Ministério da Educação, foi elaborado uma análise do texto do PPC do curso e demarcado nas DCN's os aspectos que estavam em consonância com os objetivos e diretrizes em educação. Foi evidenciado que os objetivos no PPC do curso de medicina estavam de acordo com as instruções das propostas pela DCN/Medicina (2014).

Os objetivos do curso de medicina, dentro do seu contexto educacional, têm como foco:

“à abordagem crítica, humanística e reflexiva de seus alunos com evidência na atenção primária da saúde; a competência transcultural observada no cenário real das necessidades dos locais e comunidades de atuação; a competência na gestão dos serviços de saúde nos locais de trabalho; ter conhecimento científico e técnico com habilidades de tomada de decisão em prol do coletivo em situações de urgência e emergência e, por último, ter habilidade para articular Ensino-Pesquisa e Extensão na rede de saúde com princípios de equidade, integralidade" (Rei, 2018).

Portanto, é inegável que o PPC do curso de medicina está de acordo com os objetivos e orientações das DCN's propostas em 2014 pela resolução CNE/CES n³, de 20 de junho de 2014, estabelecida pelo governo federal. 
Depois de relacionar, averiguar e apontar que o PPC do curso de medicina está de acordo com a DCN/Medicina de 2014 proposta pelo governo federal, coube ao presente estudo analisar através do modelo lógico de avaliação de programas, a evolução do processo de ensino-aprendizagem construído pelo PPC ao longo dos anos após a implantação do curso em 2014. Para isso, foram analisados o PPC de 2015/2016 e o PPC de 2018 atualizado, para uma comparação sobre o que foi atualizado e desenvolvido durante esses anos.

Assim, a análise do texto do PPC de 2018 não apresentou grandes modificações com relação ao processo de ensinoaprendizagem em comparação com o PPC de 2015/2016. No entanto, os dois documentos seguem as orientações da DCN/Medicina (2014).

Um ponto que pode ser revisto e incentivado com mais rigor no programa é se está ocorrendo o estímulo à telemedicina e às práticas de políticas de acesso às pessoas com deficiência física.

\subsection{Análise das atas do Núcleo Docente Estruturante do Curso de Medicina}

Ao analisar o PPC do curso de medicina foi identificado que este está inteiramente de acordo com a DCN proposta pelo governo federal, no entanto, o programa de avaliação CIPP destaca o planejamento do curso e verifica, de forma sistemática, todas as abordagens que precisam ser levadas em consideração, tal como o contexto, os recursos necessários para o curso ser bem-sucedido no ensino-aprendizagem, o processo de como o andamento do curso corrobora com o ensino, e o produto de todas essas informações coletadas. Para tal análise, as atas do NDE têm papel fundamental para que o caminho do curso de medicina e de outros cursos de graduação sejam coerentes com a proposta de ensino-aprendizado da instituição superior.

No estudo de Deal et al., (2018) também foi evidenciada a importância da avaliação de um programa através das análises das atas da comissão de avaliação, onde foi possível identificar dados de suporte para o desenvolvimento de planos de melhorias do programa de ensino médico avaliado.

De acordo com o portal do Ministério da Educação, o NDE foi um conceito criado pela Portaria $N^{\circ} 147$ de 2 de fevereiro de 2007, da Comissão Nacional de Avaliação da Educação Superior (CONAES), que é órgão colegiado de coordenação e supervisão do SINAES, instituído pela Lei no 10.861, de 14 de abril de 2004 (Educação, 2010). Assim, o objetivo do NDE é:

"construir o PPC do curso e assessorar o andamento do curso, avaliando e destacando pontos que necessitam de melhorias na gestão ou infraestrutura, bem como colocando à par aos outros docentes sobre mudanças que precisam ser efetivadas ao longo do curso, entre outros". (Ministério da Educação, 2010)

Por isso, é de inteira importância relacionar o PPC com o NDE e averiguar a importância destes na implementação do curso (Ministério da Educação, 2010).

Neste sentido, a análise das atas do NDE do curso de medicina foi feita a partir da primeira ata, até a última ata de cada ano, sendo os anos de implementação do curso de medicina entre os anos de 2014 a 2019.

Após a leitura das atas, foi constatado que, nos primeiros anos de vigência do curso de medicina, buscou-se estruturação do curso e do PPC, sendo que, durante o desenvolvimento do curso foram realizadas visitas técnicas semestrais da Comissão de Acompanhamento das Novas Escolas de Medicina (CAMEM), sendo que, o curso de medicina foi uma escola visitada constantemente para monitoramento de toda estruturação do curso.

Durante os anos iniciais de 2014 e 2015 foram realizadas ações de contratação, compra de equipamentos, estruturação do espaço físico e do PPC de acordo com as demandas elencadas pela CAMEM e do processo de ensino-aprendizagem. Percebe-se que o NDE foi de inteira importância para estruturação de um ensino de qualidade no curso de medicina. 
Ao longo de toda a análise das atas do NDE e colegiado, é possível perceber que todos trabalharam em prol da educação de qualidade do curso, criando propostas para um ensino-aprendizagem de qualidade, com apresentações de propostas de trabalhos de ensino, extensão e até de iniciação científica, e na qualidade do curso, com intercâmbio de ensino entre os programas de Residências Médicas. Além disso, foi verificado que o NDE e o colegiado não só atenderam aos requisitos internos do curso, mas também apoiaram os estudantes em manifestações de direito.

Com isso, é possível notar a indissociação do NDE e colegiado com o PPC e as DCNs, já que o NDE e colegiado atendem às exigências do curso para um ensino-aprendizagem sólido, com docentes e técnicos capacitados e com bons equipamentos de trabalho. O PPC aborda um ensino-aprendizagem voltado para um ensino humanístico, crítico, baseado em competências, conhecimento técnico com habilidades de tomada de decisão. Por fim, a DCN traz consonância para o norteamento do curso como, abordagem crítica, competência transcultural, competência na gestão dos serviços, ter conhecimento científico e habilidades para articular ensino-pesquisa e extensão. (Educação, 2014).

\subsection{Metodologias Ativas de Ensino no Curso de Medicina}

As metodologias ativas são modelos de aprendizagem que viabilizam o acesso do aluno às práticas educacionais, bem como o orientam para, de fato, realizar tais práticas em sua profissão. A palavra ativa já propõe algo que passe do plano teórico para a real prática dentro da comunidade a qual o aluno irá se deparar, ou seja, o aluno é o principal protagonista nas realizações de práticas e de aprendizados adquiridos no modelo de metodologia passiva em que o docente é o protagonista do ensino. São nestes moldes ativos que o estudante irá colocar todo o seu conhecimento em ação, além da experiência adquirida em saber lidar com algo que possa vir a ser necessário e imediato na tomada de decisões (Mitre et al., 2012).

Nesse sentido, o PPC traz alguns parâmetros e modelos de metodologias ativas que funcionam no curso de medicina. São quatro as principais modalidades de ensino/aprendizagem que são adotadas no PPC do curso de medicina - Aprendizagem Baseada em Problemas (ABP / PBL) e Aprendizagem baseada em equipe (ABE/ TBL); Problematização; Pedagogia de projetos - Aprendizagem Baseada em Projetos (ABP/PBjL); Ação- Reflexão- Ação. Essas são metodologias utilizadas por diversos autores para trabalhar a tomada de decisão, raciocínio crítico e reflexivo, comunicação e engajamento em equipe (Almeida e Batista, 2011).

\subsection{Análise qualitativa das entrevistas com os atores envolvidos no projeto}

Foram realizadas as análises das respostas das entrevistas de 14 (quatorze) participantes, sendo, 2 técnicos, 4 discentes e 8 docentes. As análises das respostas serão apresentadas separadamente, seguindo a ordem dos técnicos, discentes e docentes, respectivamente.

\subsubsection{Entrevistas com os técnicos do curso de medicina}

Os técnicos que responderam a entrevista pertencem a setores distintos de atuação, evidenciando um olhar diferenciado para as questões do curso de medicina. O primeiro atua como preceptor da rede de saúde do munícipio e o segundo atua dentro do laboratório de ensino na instituição. Diante disso, depreende-se que o técnico 1 considera que é importante uma centralização dos estágios no município para que seja melhor organizado, bem como, pontua que a estrutura de serviços precisa ser melhorada.

O técnico 2 aponta que a estrutura é boa para o aprendizado teórico e prático dos discentes. Nesse sentido, foi percebido que o técnico que atua na rede de saúde do município reivindica por melhorias estruturais e administrativas nos serviços de saúde da rede, enquanto o técnico 2 está satisfeito com a infraestrutura onde atua e é relativamente nova. Além disso, ambos autores consideram o PPC adequado para o ensino dos discentes. 


\subsubsection{Entrevistas com os discentes do curso de medicina}

O corpo discente do curso de medicina também avaliou o PPC, a gestão do curso e a infraestrutura. Diante dos dados coletados, foi destacado que gostam da proposta do PPC, mas que necessita de adaptações e melhorias, como a carga horária de algumas unidades curriculares. Isso foi evidenciado na fala de um dos estudantes:

"Gosto, acho que tem algumas falhas, como pouca carga horária de radiologia e ausência de uma disciplina teórica de patologia clínica. Além disso, é preciso uma reorganização de algumas disciplinas que foram mais contempladas com uma carga horária maior, como a disciplina de gestão em saúde e psicologia em saúde, onde a carga horária é bem superior que cirurgia geral, infectologia e patologia clínica, por exemplo”. (Estudante 2).

Sobre as questões administrativas da instituição, os estudantes relataram que poderiam ocorrer melhorias na comunicação entre os setores administrativos, destacando que:

"O Curso necessita de mais funcionários para atenderem a demanda". (Estudante 1).

\subsubsection{Entrevistas com os docentes do curso de medicina}

Entre o corpo docente foram apresentadas questões sobre o PPC, as metodologias de ensino utilizadas e o atendimento em saúde quanto à demanda da região. Os resultados foram quase unânimes. Em geral, a maioria aprova a proposta pedagógica do curso, embora ressaltem que seja necessária uma reorganização das disciplinas e uma execução plena da proposta, como mostra o recorte de alguns entrevistados.

\footnotetext{
"Gosto da proposta, pois utiliza de métodos ativos de ensino-aprendizagem" (Docente 1)

"O aluno acaba sobrecarregado com a forma das disciplinas serem dadas em módulos. Se fosse ao longo do semestre o aluno teria melhor aproveitamento do conteúdo e principalmente das aulas práticas." (Docente 3)

"Acho a proposta excelente, embora seja difícil executá-la de forma plena". (Docente 4)
}

Quando foi perguntado se conseguem atender aos objetivos de ensino da proposta do curso, a maioria respondeu que consegue, e pontuaram que utilizam metodologias ativas de ensino adequadas para o processo de ensino-aprendizado dos estudantes.

As perguntas também foram direcionadas para o papel da universidade no município que está inserido e foi relatado pelos docentes que o papel da universidade é importante dentro do município. Este contexto foi evidenciado nas falas dos docentes.

"A presença da Universidade dentro dos serviços de saúde apresenta melhorias diretas e indiretas na Atenção à Saúde como capacitações para os profissionais da rede, educação em saúde para os usuários, novos tipos de assistência e melhora na produtividade, mudanças na gestão dos serviços.” (Docente 2)

De acordo com Bicas e Kornis (2021):

"os sistemas de avaliação do ensino médico dependem da articulação política dos atores envolvidos, da força das demandas sociais sobre o tema e do equilíbrio entre as prioridades governamentais de expansão do ensino médico e regulação de sua qualidade." 
Existem vários modelos de avaliação do programa de ensino em saúde (Goldie, 2006; Bracialli \& Oliveira, 2012, Lira \& Nogueira, 2016). No estudo de Casas et al. (2017) não foi utilizado nenhum modelo de avaliação específico, no entanto, foi utilizado o Structured Clinical Examinations (OSCE) para avaliação das competências médicas adquiridas entre os estudantes de medicina e avaliou a interferência da empatia nas habilidades médicas do curso. No presente estudo foi demonstrado que o modelo lógico ou CIPP foi eficaz na avaliação do curso de medicina da instituição de ensino avaliada.

Assim, o presente artigo teve como objetivo mostrar a importância do programa de avaliação do modelo lógico para identificar as potencialidades e fragilidades do curso de medicina proporcionando informações para mudanças necessárias no que diz respeito à busca pela melhoria do ensino-aprendizagem do curso. Sendo que, o estudo das DCN's do Curso de Medicina, do PPC, do NDE e colegiado do curso foram parâmetros importantes de análise nesse processo de investigação do programa de medicina. Investigar estes documentos e compará-los permitiu uma visão ampla do funcionamento do curso, assim como, apontar os pontos positivos e os que precisam se readequados à realidade do curso e comunidade.

\section{Conclusão}

Com este estudo, percebe-se que o curso de medicina por ser relativamente novo, é um curso que está sempre buscando uma forma de planejamento que forneça a seus estudantes um bom ensino, bem como sempre proporcionando novas formas de fazer educação como projetos de extensão, pesquisa e projetos de educação pelo trabalho voltados para a sociedade; tudo isso com profissionais capacitados em atuar no processo de formação de profissionais. Ademais, é importante destacar que, diante de todas as análises das atas do NDE e colegiado, a instituição e o corpo docente buscam atender às necessidades de seus alunos no que diz respeito a disponibilização de materiais e equipamentos que fomentam o ensino, o que demonstra preocupação com a formação dos estudantes.

Todos os aspectos do programa do curso de medicina foram analisados sob a ótica do modelo lógico de avaliação, tendo como resultado vários apontamentos que sinalizam para a necessidade de uma restruturação de aspectos que precisam ser melhorados, no entanto, é visível que o curso está no caminho certo para atingir um ensino de qualidade cada vez mais sólido na instituição.

Sugere-se que mais estudos sejam realizados utilizando este modelo lógico com o intuito de fornecer subsídios de melhorias para seus programas e direcionar uma avaliação interna de qualidade.

\section{Agradecimentos}

Agradecemos à Pró-Reitoria de Pesquisa (PROPE) e o Departamento e Curso de Medicina, do Campus Dom Bosco, da Universidade Federal de São João del-Rei (UFSJ). Agradecemos também à FAPEMIG pelo financiamento da bolsa de pesquisa para a autora.

\section{Referências}

Andrade, C. B., \& Monteiro, M. I. (2007). Envelhecimento e capacidade para o trabalho dos trabalhadores de higiene e limpeza hospitalar. Revista da Escola de Enfermagem da USP, 41, 237-244.

AbbadI, G. d., Souza, I. D. B., LavalII, A. d., \& SouzaI, S. C. (2012). Modelos lógicos em avaliação de sistemas instrucionais: dois estudos de casos. Revista Psicologia, Organizações e Trabalhos. 12, 2, 185-202.

Almeida, M. T. C, Batista, N. A. Ser docente em métodos ativos de ensino-aprendizagem na formação do médico. Revista Brasileira de Educação Médica, 35(4):468-476,2011.

Braccialli, L. A. D. \& Oliveira, M. A. C. (2012). Desafios na formação médica: a contribuição da avaliação. Revista Brasileira de Educação Médica. 36, 2, $268-280$.

Bicas, R. B. S. \& Kornis, G. E. M. (2021). Avaliação globral do ensino médico brasileiro: interesses dos atores envolvidos. Edud. Aval. Educ. 32 , 1-25. 
Research, Society and Development, v. 11, n. 2, e25911225895, 2022

(CC BY 4.0) | ISSN 2525-3409 | DOI: http://dx.doi.org/10.33448/rsd-v11i2.25895

Casas, R. S., Xuanb, Z., Jacksona, A. H., Stanfielda, L. E., Harveyc, N. C. \& Chena, D. C. (2017) Associations of medical student enpathy with clinical competence. Patient Education and Counseling. 100, 742-747.

Castro, V. R. (2014). Avaliação de programas educacionais nas profissões da saúde: conceitos básicos. Tópicos fundamentais para a formação e o desenvolvimento docente para professores dos cursos da área da saúde, (p. 11). Ribeirão Preto.

Deal, S. B., Seabott, H., Chang, L. \& Alseidi, A. A. (2018). The Program Evaluation Committee in Action: Lessons Learned From a General Surgery Residency's Experience. Journal of Surgical Education. 75, 1, 7-13.

Educação, C. N. (2014). Diretrizes Curriculares Nacionais Medicina de 2014. Fonte: Faceres: https://faceres.com.br/cursos/medicina/diretrizes-curricularesnacionais-medicina-de-2014. Acesso em: 08/09/2021

Educação, M. d. ( 2010). Parecer CONAES $N^{\circ} 4$ de 17 junho de 2010, sobre o Núcleo Docente Estruturante. Portal Ministério da Educação: http://portal.mec.gov.br/index.php?option=com_docman\&view=download\&alias=6884-parecer-conae-nde4-2010\&category_slug=outubro-2010pdf\&Itemid=30192. Acesso em: 08/09/2021

Educação, O. P. (2018). O que são e para que servem as diretrizes curriculares? Todos pela educação: https://www.todospelaeducacao.org.br/conteudo/o-quesao-e-para-que-servem-as-diretrizes-curriculares-/. Acesso em: 08/09/2021

Frye, A. W., \& Hemmer, P. A. (2012). Program evaluation models and related theories: AMEE Guide No. 67. Journal Medical Teacher, 288-299.

Haji, F., Morin, M.P. \& Parker, K. (2013). Rethinking programme evaluation in health professions education: beyond 'did it work?'. Med. Educ., 342-51.

Lira, G. V., \& Nogueira, D. N. (2016). Teoria e método para um modelo de avaliação dos hospitais de ensino do Brasil. Revista Brasileira de Educação Médica. 40, 1, 4-10.

Mendonça, E. F. (2014). Diretrizes Curriculares Nacionais Medicina. FACERES: https://faceres.c/om.br/cursos/medicina/diretrizes-curriculares-nacionaismedicina-de-2014.

Mezzalira, D. P., Ferreira, A. C., Andrade, G. H., Arruda, C. R. P. \& Mattia, B. J. (2022). A humanização na educação médica no Brasil. Research, Society and Development. 11, 1, e57711125337.

Mitre, S. M., Siqueira-Batista, R., Girardi-de-Mendonça, J. M., Morais-Pinto, N. M., Meirelles, C. A. B., Pinto-Porto, C., Moreira, T., Hoffmann, L. M. A. (2012). Metodologias ativas de ensino-aprendizagem na formação profissional em saúde: debates atuais. Ciências Saúde Coletiva. 13(2):2133-44.

Rei, U. F. (2018). Projeto Pedagógico do Curso de Graduação em Medicina. Minas Gerais, Brasil.

Social, A. D. (2018). Homologação das Diretrizes Curriculares Nacionais pelo ministro começa a mudar o ensino médio. Ministério da Educação: http://portal.mec.gov.br/component/tags/tag/50361-den. t 